\title{
Telomere Length in the Newborn
}

\author{
KOJI OKUDA, ARLENE BARDEGUEZ, JEFFREY P. GARDNER, PAULETTE RODRIGUEZ, $\dagger$ \\ VIJAYA GANESH, MASAYUKI KIMURA, JOAN SKURNICK, GIRGIS AWAD, AND \\ ABRAHAM AVIV \\ The Hypertension Research Center (K.O., J.P.G., M.K., G.A., A.A.), Department of OB/GYN and Women's \\ Health (A.B., P.R., V.G.), Department of Preventive Medicine and Community Health (J.S.), University of \\ Medicine and Dentistry of New Jersey, New Jersey Medical School, Newark, New Jersey
}

\begin{abstract}
ABS
Telomere length is similar in different organs of the human
fetus but variable among fetuses. During extrauterine life telo-
mere length is highly variable among individuals and longer in
women than men. In the present work we addressed the follow-
ing questions: 1) Are there sex-related differences in telomere
length at birth? 2) Is there synchrony (i.e. correlation in length)
of telomeres in tissues within the newborn? 3) Is the variability
in telomere length among newborns as large as that in adults? We
studied normal male and female newborns who donated DNA
samples from three sources: white blood cells, umbilical artery,
and foreskin. Telomere length was measured by the mean length
of the terminal restriction fragments (TRF). TRF length was not
different between male and female newborns. It was highly
synchronized among the DNA samples from white blood cells,
umbilical artery and skin within individual donors but exhibited
a high variability among donors. We conclude that there is no
\end{abstract}
evidence for the effect of sex on telomere length at birth, suggesting that longer telomeres in women than men arise from a slower rate of telomeric attrition in women. The variability in telomere length among newborns and synchrony in telomere length within organs of the newborn are consistent with the concept that variations in telomere length among adults are in large part attributed to determinants (genetic and environmental) that start exerting their effect in utero. (Pediatr Res 52: 377-381, 2002)

\section{Abbreviations}

UMDNJ, University of Medicine and Dentistry of New Jersey TRF, terminal restriction fragments

UA, umbilical artery

WBCs, white blood cells
The loss of telomere repeats associated with replication of human somatic cells in culture is central to the concept that in these cells telomeres serve as a "mitotic clock" (1). In human beings, telomere length of replicating somatic cells is inversely related to donor age (2-4), highly variable among donors of the same age $(2-4)$, highly heritable $(3,5)$, and longer in women than in men $(5,6)$. These observations suggest that in human somatic cells telomere length is a biomarker of replicative history not only in vitro but also in vivo and that it is modified by genetic factors and sex.

In contrast to the considerable information available about telomere length in humans during extrauterine life, little is known about telomere length during intrauterine life. Limited

October 12, 2001; November 30, 2001.

Correspondence: Abraham Aviv, Room F-464, MSB, Hypertension Research Center, University of Medicine and Dentistry of New Jersey, 185 South Orange Avenue, Newark, New Jersey 07103-2714; e-mail: avivab@umdnj.edu

$\dagger$ Dr. Paulette Rodriguez was recently killed in an automobile accident. This paper is dedicated to her memory.

This work was supported by a grant from the New Jersey Health Foundation. K. Okuda was supported by a post-doctoral fellowship of the American Heart Association, Heritage Foundation.

DOI: 10.1203/01.PDR.0000022341.72856.72 information indicates that in utero, the length of human telomeres is highly synchronized in that it is similar among tissues of the same fetus, but variable among fetuses (7). The present research was undertaken to answer the following key questions about telomere length: 1) Do male and female newborns differ in the length of their telomeres? 2) At birth, is there synchrony of telomere length in cells derived from three tissues (blood, umbilical artery and foreskin)? 3) Is the variability of telomere length among newborns the same as that observed in adults?

\section{METHODS}

Subjects. We studied normal newborns born at two hospitals, i.e. The University Hospital of the University of Medicine and Dentistry of NJ (UMDNJ) in Newark, NJ, and the affiliated Hackensack University Medical Center in Hackensack, NJ. General characteristics of the newborns, including maternal age, parity and gravidity, birth weight and gestational age are presented in Tables 1 and 2. Twenty-seven mothers were diagnosed with hypertension during pregnancy, defined by blood pressure equal to or greater than $140 / 90 \mathrm{~mm} \mathrm{Hg}$, without clinical evidence of edema or proteinuria, after $20 \mathrm{wk}$ of gestation. Seventeen mothers were diagnosed as having pre- 
Table 1. General characteristics of study population

\begin{tabular}{lc}
\hline Blacks/Whites/Hispanics/Others* & $86 / 48 / 29 / 5$ \\
Male/Female & $85 / 83$ \\
Gestational Age (weeks) & $38.57 \pm 0.14$ \\
& $(32-42)$ \\
Weight (gms) & $3235 \pm 41$ \\
& $(1595-4745)$ \\
Mother's age (years) & $26.5 \pm 0.53$ \\
& $(15-43)$ \\
Parity/Gravidity & $1 / 0(0-7) / 2.8(1-12)$ \\
\hline
\end{tabular}

* Others include newborns whose ethnicity was undefined.

Brackets denote range.

eclampsia, defined by blood pressure equal to or greater than 140/90 mm Hg plus clinical evidence of edema, proteinuria, or both, after 20 wk of gestation. Seven mothers had pregestational diabetes. The following exclusion criteria were implemented for mothers: known HIV-infection or unknown HIVserostatus, positive hepatitis serology, intrauterine infection, history of any chronic infection, autoimmune or metabolic diseases other than diabetes, history of chronic heart lung and kidney diseases, history of sickle cell anemia and cancer, history of drug abuse immediately before and during pregnancy. Newborns with chromosomal or any other major congenital defects were also excluded. We abstracted from the medical record of each newborn the following information: maternal race, mode of delivery, infant gender, Apgar scores, birthweight. Gestational age was computed from the estimated date of confinement (EDC) and the actual birth date, which were obtained from the medical charts. This research was approved by the Institutional Review Board of the UMDNJ and Hackensack University Medical Center. Informed consent was obtained from mothers.

Specimens. Immediately after birth, $10 \mathrm{~mL}$ of umbilical cord blood was collected and a $20 \mathrm{~cm}$ segment of the umbilical cord placed in ice-cold sterile PBS (PBS) with antibiotics. The umbilical artery was removed and stripped of the adherent adventrical tissue. Foreskin specimens were obtained from a subset of male newborns undergoing circumcision. No skin specimens were available from white newborns. These specimens were immediately immersed in ice-cold sterile PBS plus antibodies. Erythrocytes in blood specimens were lysed with a hypotonic solution, specimens centrifuged and pellets (primarily comprising white blood cells) and tissue specimens washed three times with PBS. All samples were stored at $-80^{\circ} \mathrm{C}$ until DNA extraction. A complete set of specimens (white blood cells and umbilical artery cells for newborn girls; white blood cells, umbilical artery and skin cells for newborn boys) was not available from all donors.

DNA isolation and measurements of terminal restriction fragmented (TRF) length. In preparation for DNA extraction, solid tissues were immersed in liquid nitrogen and specimens ( $\sim 50 \mathrm{mg}$ each) were ground in an alloy tool steel mortar and pestle set (Fisher, Pittsburgh, PA, U.S.A.). Ground tissues or white blood cell pellets were suspended in $0.5 \mathrm{~mL}$ buffer $(\mathrm{NaCl}$ $100 \mathrm{mM}$, Tris $10 \mathrm{mM}$, SDS 1.0\%, EDTA $25 \mathrm{mM}, \mathrm{pH} \mathrm{8.0)}$ and specimens were thawed at $50^{\circ} \mathrm{C}$. Proteinase $\mathrm{K}(0.2 \mathrm{mg} / \mathrm{mL})$ was added and mixture digested overnight. DNA was extracted with phenol/chloroform/isoamyl alcohol and with chloroform/ isoamyl alcohol, precipitated and dissolved in $10 \mathrm{mM}$ Tris- $\mathrm{HCl}$ (pH 8.0) and $1 \mathrm{mM}$ EDTA. TRF measurements were performed as previously described using telomeric probe (digoxigenin 3'- end labeled 5'-(CCTAAA $\left.)_{3}\right)(4,5)$. The probe was detected by the digoxigenin luminescent detection procedure (Boehringer Mannheim, Indianapolis, IN, U.S.A.) and exposed on x-ray film.

To determine TRF length, each lane was scanned between 5 and $20 \mathrm{~kb}$ with a densitometer (Molecular Dynamics, Image Quant) and compared with the closest of four reference molecular weight ladders on each gel. The length of TRFs was calculated as previously described (7) using the formula:

$$
\mathrm{TRF}=\Sigma \mathrm{OD}(i) / \Sigma(\mathrm{OD}(i) / \mathrm{MW}(i),
$$

where $\mathrm{OD}(i)=\mathrm{OD}$ at a given position and $\mathrm{MW}(i)=$ molecular mass at the same position. This formula accounts for the fact that longer telomeres bind more of the labeled probe and consequently appear darker on the x-ray film. TRF length refers to the average TRF length for a sample run in triplicate on different gels.

Statistical analysis. TRF lengths, birth weights and gestational age of Blacks, Hispanics and Whites were compared by ANOVA. Comparisons of TRF lengths by gender, occurrence of hypertension during pregnancy, preeclampsia and pregestational diabetes were performed by $t$ tests. Synchrony, i.e. equality in TRF length among tissues (cells) from the same infant, was evaluated by two methods. First, the mean differences between blood, umbilical artery and foreskin TRF lengths were obtained for each newborn, and mean differences were compared with 0 (no difference) by paired $t$ tests. Second, least-square linear regression lines were estimated for TRF lengths from blood as a function of umbilical artery and foreskin TRF lengths. Under the premise of equality, the slope would be 1 and the intercept 0 . F tests with 2 degrees of

Table 2. Birth weights and gestational ages of newborns

\begin{tabular}{lcc}
\hline Group & $\begin{array}{c}\text { Birth Weights (gms) } \\
\text { Mean } \pm \mathrm{SD}\end{array}$ & $\begin{array}{c}\text { Gestational Age (weeks) } \\
\text { Mean } \pm \mathrm{SD}\end{array}$ \\
\hline Blacks & $3120 \pm 57^{*}$ & $38.5 \pm 0.21$ \\
& $(\mathrm{n}=86)$ & $(\mathrm{n}=85)$ \\
Whites & $3413 \pm 74$ & $38.6 \pm 0.23$ \\
& $(\mathrm{n}=48)$ & $(\mathrm{n}=48)$ \\
Hispanics & $3282 \pm 77$ & $38.6 \pm 0.29$ \\
& $(\mathrm{n}=29)$ & $(\mathrm{n}=29)$ \\
\hline
\end{tabular}

* Statistically significant at $p=0.0016$ compared with Whites $(t$ for contrast $=3.20, \mathrm{df}=160$ ).

Table 3. TRF length

\begin{tabular}{cccc}
\hline Group & WBC $(\mathrm{kb})$ & UA $(\mathrm{kb})$ & Foreskin $(\mathrm{kb})$ \\
\hline Blacks & $10.98 \pm 0.086$ & $10.99 \pm 0.081$ & $10.91 \pm 0.160$ \\
& $(n=83)$ & $(n=83)$ & $(n=21)$ \\
Whites & $10.92 \pm 0.110$ & $10.89 \pm 0.094$ & $(n=0)$ \\
& $(n=48)$ & $(n=43)$ & \\
Hispanics & $11.26 \pm 0.104$ & $11.25 \pm 0.115$ & $11.07 \pm 0.225$ \\
& $(n=29)$ & $(n=27)$ & $(n=2)$ \\
Overall & $11.01 \pm 0.058$ & $11.01 \pm 0.055$ & $10.92 \pm 0.147$ \\
& $(n=165)$ & $(n=158)$ & $(n=23)$ \\
\hline
\end{tabular}

WBC, white blood cells; UA, umbilical artery. 


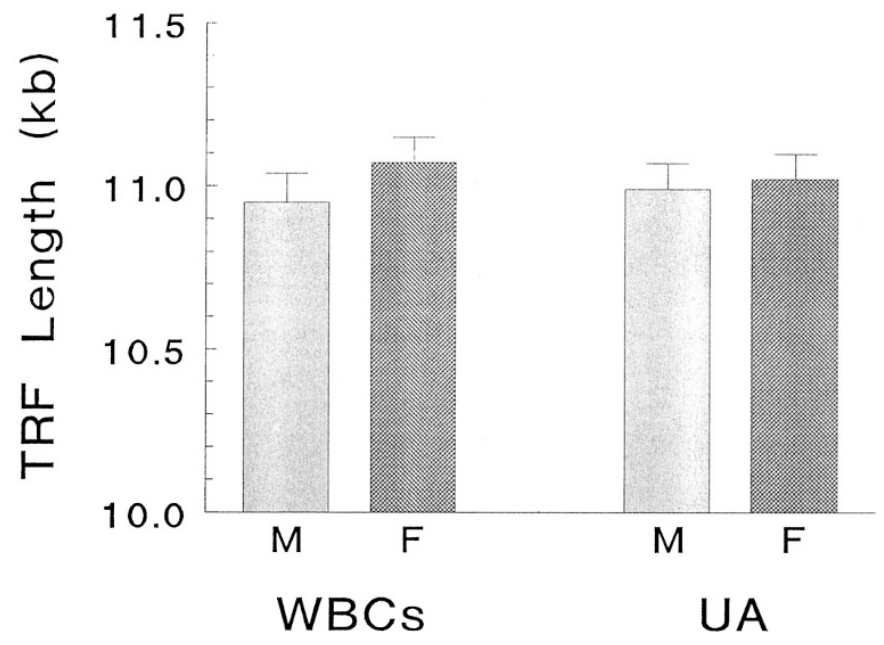

Figure 1. TRF lengths in white blood cells (WBC) and cells from the umbilical artery (UA) in male and female newborns.

freedom in the numerator were run to test the pair of parameter estimates against these values. To evaluate the scatter of the distribution of TRF lengths in infants, $95 \%$ confidence intervals based on the $\chi^{2}$ distribution were constructed for the point estimates of variance of TRF lengths in blood and in umbilical artery, after checking the approximate normality of the distributions. A multiple linear regression of TRF length as a function of birth weight and gestational age was performed to evaluate possible correlation of birth weight with TRF length, after adjustment for gestational age. The strength of association between TRF lengths and age, parity and gravity was measured by linear and rank correlation coefficients. Analyses were conducted using SAS software (SAS ${ }^{\circledR}$, Durham, NC, U.S.A.). The criterion of statistical significance was two-tailed $p<$ 0.05 . Data are presented as mean $\pm \mathrm{SEM}$, and $p$ values are two-tailed, unless specified otherwise.

\section{RESULTS}

Table 2 presents the birth weights and gestational ages of the newborns as characterized by ethnicity. The birth weight of Blacks was significantly lower than that of Whites $(t=3.20, p$ $=0.0016)$. There were no statistically significant differences in TRF length among the three ethnic groups (Table 3). There were also no sex related differences in TRF length in white blood cells $($ males $=10.95 \pm 0.088 \mathrm{~kb}$; females $=11.07 \pm$
$0.077 \mathrm{~kb}, t=1.00, p=0.3$ ) and in umbilical artery cells $($ males $=10.99 \pm 0.078 \mathrm{~kb}$; females $=11.02 \pm 0.077 \mathrm{~kb}, t=$ $0.29, p=0.8$ ) (Fig. 1).

Table 4 summarizes data about TRF lengths in subsets of newborns whose mothers were diagnosed with hypertension during pregnancy, preeclampsia and pregestational diabetes. There were no differences in TRF lengths in WBCs or umbilical artery cells between these newborns and newborns of mothers who did not exhibit those complications.

Strong similarity of TRF length was observed for white blood cells and umbilical artery cells; the mean difference in TRF lengths was not significantly different from $0(0.013 \pm$ $0.027 \mathrm{~kb} ; t=0.47, p=0.64 ; n=155)$. There was also no difference between TRF length of skin cells and umbilical artery cells (mean difference $=0.095 \pm 0.058 \mathrm{~kb} ; t=1.63, p$ $=0.12 ; n=22$ ). However, the TRF lengths of skin cells were significantly longer than TRF lengths of white blood cells (mean difference $=0.210 \pm 0.054 \mathrm{~kb} ; t=3.87, p=0.001 ; n$ $=20$ ). To further check for synchrony in TRF length among cells from the newborns, TRF length of white blood cells was plotted against TRF length in umbilical artery cells (Fig. 2A). The TRF lengths of white blood cells and umbilical artery cells were highly correlated $(r=0.89, p=0.0001)$. Furthermore, the least-squares line was consistent with the hypothesis of line of identity: white blood cells $=0.007+0.989$ umbilical artery cells (test of 0 intercept and slope of $1: p=0.10$ ). A subset of male infants had specimens from skin and umbilical artery cells or skin and white blood cells. In these newborns strong correlations were observed in the regressions of TRF length in skin cells on TRF length in white blood cells $(r=0.97, p=$ $0.0001)$ and on TRF length in umbilical artery cells $(r=0.94$, $p=0.0001$ ) (Fig. 2B,C). However, the slopes and intercepts of these regressions were different from 1 and 0 , respectively. The least-squares lines were as follows: skin cells $=2.28+$ 0.80 white blood cells $(p=0.0001)$, and skin cells $=2.18+$ 0.8 umbilical artery cells $(p=0.009)$.

The ranges and variance in TRF lengths of the cells were as follows: For white blood cells range $=8.55-13.32 \mathrm{~kb}$, variance $=0.50 \mathrm{~kb}^{2}$ with a $95 \%$ confidence interval of $0.46 \mathrm{~kb}^{2}, 0.71$ $\mathrm{kb}^{2}$; for umbilical artery cells, range $9.02-12.59 \mathrm{~kb}$, variance $=$ $0.47 \mathrm{~kb}^{2}$, with $95 \%$ confidence interval of $0.38 \mathrm{~kb}^{2}, 0.59 \mathrm{~kb}^{2}$. For skin cells, range $=9.26-12.32 \mathrm{~kb}$ and variance of 0.50 $\mathrm{kb}^{2}$; the sample size of 23 did not allow for a reliable confidence interval about the variance. In our previous study,

Table 4. TRF length of newborns whose mothers had hypertension during pregnancy, preeclampsia, and pregestational diabetes

\begin{tabular}{lcc}
\hline \multicolumn{1}{c}{ Diagnosis } & WBC $(\mathrm{kb})$ & $\mathrm{UA}(\mathrm{kb})$ \\
\hline Hypertension during pregnancy & $11.04 \pm 0.136(n=27)$ & $10.89 \pm 0.136(n=24)$ \\
No hypertension during & $11.00 \pm 0.065(n=138)$ & $11.03 \pm 0.060(n=134)$ \\
pregnancy & $(p=0.81)$ & $(p=0.37)$ \\
Preeclampsia & $11.18 \pm 0.183(n=17)$ & $11.11 \pm 0.192(n=15)$ \\
No preeclampsia & $10.99 \pm 0.062(n=148)$ & $11.00 \pm 0.057(n=143)$ \\
Pregestational diabetes & $(p=0.33)$ & $(p=0.55)$ \\
No pregestational diabetes & $10.89 \pm 0.148(n=7)$ & $10.59 \pm 0.218(n=7)$ \\
& $11.02 \pm 0.061(n=158)$ & $11.03 \pm 0.056(n=158)$ \\
& $(p=0.66)$ & $(p=0.10)$
\end{tabular}

WBC, white blood cells; UA, umbilical artery. 
examining TRF lengths in white blood cells of adults (6), the variance after age-adjustment was $0.63 \mathrm{~kb}^{2}$; after adjustment for gender as well as the variance was $0.62 \mathrm{~kb}^{2}$. These variance estimates for adults lie within the $95 \%$ confidence interval for variance of TRF lengths in white blood cells of infants.

There was no significant relation of TRF lengths from blood or umbilical artery cells with birthweight, gestational age, and

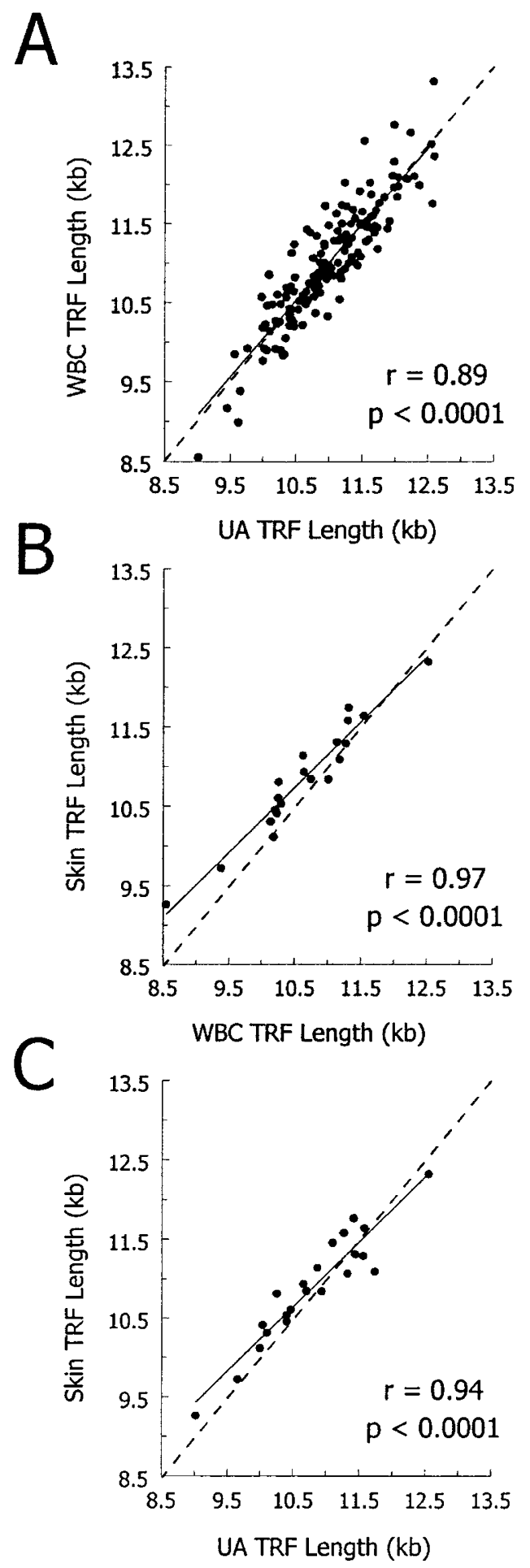

Figure 2. Correlations among TRF lengths in white blood cells (WBC) and cells from the umbilical artery (UA) and skin. Interrupted lines denote lines of identity. birthweight adjusted for gestational age. There was also no significant correlation of TRF lengths with gravidity or parity. Maternal age was positively correlated with TRF length in white blood cells. Although weak, the correlation was statistically significant $(r=0.18, p=0.02)$.

\section{DISCUSSION}

The main findings of this work are: 1) There are no differences between male and female newborns in TRF length. 2) At birth, TRF length was highly synchronized among white blood cells, umbilical artery cells and skin cells. 3) Variations in TRF length among newborns are as wide as variations in TRF lengths among adults. The implications of these findings are discussed below.

Two recent studies have found that after age-adjustment, TRF length, as determined in white blood cells, was longer in women than men $(5,6)$. The equal TRF length in male and female newborns suggests that sex-related differences in TRF length arise from different rates of telomere attrition during extrauterine life. The most likely reason for this effect is estrogen. The effect of estrogen on telomere attrition during extrauterine life may be exerted in two ways. First, estrogen can stimulate telomerase, given that an estrogen response element exists on the catalytic subunit of the enzyme (8). Telomerase is a reverse transcriptase that counters telomeric attrition by adding back telomeric repeats onto the ends of chromosomes $(9,10)$. Second, estrogen reduces oxidative stress $(11,12)$, and reactive oxygen species accelerate the rate of telomere attrition (13).

The present study extends previous observations of synchronization in telomere length among multiple organs of the human fetus (7). This synchronization was also observed in telomere length from three different tissues in the newborns. We note, however, that based on a limited number of skin samples in male newborns, telomere length in skin specimens was significantly shorter in skin than in white blood cells. The biologic meaning of this difference is not clear. What is clear, however, is that variations in telomere length among individuals far exceed variation in telomere length in different tissues within the same individual. To some extent, this phenomenon is observed not only during intrauterine life and at birth but also throughout extrauterine life (14-16). Since variations in telomere length among individuals are to a large part genetically based, the effect of genetic determinants on telomere length may be already exerted in utero $(3,5)$.

A recent study reported that TRF length in umbilical cord blood cells was significantly longer in "very low birthweight" preterm compared with "low birthweight" neonates (17). These findings need confirmation, given that the conclusion of the study was based on 13 newborns and the considerable interindividual variation in TRF length observed at birth.

Variability in telomere length among individuals may arise from variation in telomere length at birth, variation in the rate of telomere attrition thereafter, or both. The present study shows that variability in telomere length among newborns is as large as that observed in later life $(2-4,6,18)$. This finding does not exclude the possibility that the pace of telomere 
attrition is different among persons during extrauterine life, since data showing age-dependent telomere attrition are derived from cross-population studies with little information available from prospective studies examining age-dependent telomere attrition in individual subjects.

Recent studies have found intriguing associations between telomere length and biologic indicators of vascular aging $(5,6$, $15,19)$. The ramifications of these studies are unknown at present. If telomeres serve as biologic timekeepers in human beings, understanding the determinants that contribute to the variability in telomere length during intrauterine life will provide an important insight not only into developmental biology of the newborn but also the related biology of human aging.

Acknowledgments. The authors thank Nadine Powers for her secretarial help, Keith Ganesh for his technical assistance and data management, and Dr. Manuel Alvarez for his assistance with patient enrollment.

\section{REFERENCES}

1. Harley CB, Vaziri H, Counter CM, Allsopp RC 1992 The telomere hypothesis of cellular aging. Exp Gerontol 27:375-382

2. Vaziri H, Schachter F, Uchida I, Wei L, Zhu X, Effros R, Cohen D, Harley CB 1993 Loss of telomeric DNA during aging of normal and trisomy 21 human lymphocytes. Am J Hum Genet 52:661-667

3. Slagboom PE, Droog S, Boomsma DI 1994 Genetic determination of telomere size in humans: A twin study of three age groups. Am J Hum Genet 55:876-882

4. Okuda K, Khan MY, Skurnick J, Kimura M, Aviv H, Aviv A 2000 Telomere attrition of the human abdominal aorta: relationship with age and atherosclerosis. Atherosclerosis 152:391-398
5. Jeanclos E, Schork NJ, Kyviv KO, Kimura M, Skurnick JH, Aviv A 2000 Telomere length inversely correlates with pulse pressure and is highly familial. Hypertension 36:195-200

6. Benetos A, Okuda K, Lajemi M, Kimura M, Thomas F, Skurnick J, Labat C, Bean K, Aviv A 2001 Telomere length as indicator of biologic aging: the gender effect and relation with pulse pressure and pulse wave velocity. Hypertension 37(part 2):381385

7. Youngren K, Jeanclos E, Aviv H, Kimura M, Stock J, Hanna M, Skurnick J, Bardeguez A, Aviv A 1998 Synchrony in telomere length of the human fetus. Hum Genet 102:640-643

8. Kyo S, Takakura M, Kanaya T, Zhuo W, Fujimoto K, Nishio Y, Orimo A, Inoue M 1999 Estrogen activates telomerase. Cancer Res 59:5917-5921

9. Engelhardt M, Martens UM 1998 The implication of telomerase activity and telomere stability for replicative aging and cellular immortality (Review) Oncol Rep 5:10431052

10. Morin GB 1989 The human telomere terminal transferase enzyme is a ribonucleoprotein that synthesizes TTAGGG repeats. Cell 59:521-529

11. Tang M, Subbiah MT 1996 Estrogens protect against hydrogen peroxide and arachidonic acid induced DNA damage. Biochim Biophys Acta 1299:155-159

12. Romer W, Oettel M, Menzenbach B, Droescher P, Schwarz S 1997 Novel estrogens and their radical scavenging effects, iron-chelating and total antioxidative activities: 17 alpha-substituted analogs of delta 9 (11)-dehydro-17 beta estradiol. Steroids 62:688-694

13. von Zglinicki T 2000 Role of oxidative stress in telomere length regulation and replicative senescence. Ann NY Acad Sci 908:99-110

14. Martens UM, Zijlmans JM, Poon SS, Dragowska W, Yui J, Chavez EA, Ward RK, Lansdorp PM 1998 Short telomeres on human chromosome 17p. Nat Genet 18:76-80

15. von Zglinicki T, Serra V, Lorenz M, Saretzki G, Lenzen-Grossimlighaus R, Gressner R, Risch Steinhagen-Thiessen E 2000 Short telomeres in patients with vascular dementia: An indicator of low antioxidative capacity and possible risk factor? Lab Invest 80:1739-1747

16. Butler MG, Tilburst J, DeVeries A, Muralidher B, Aul G, Hedges L, Atkinson J, Schwartz H 1998 Comparison of chromosome telomere integrity in multiple tissues from subjects at different ages. Cancer Genet Cytogenet 105:138-144

17. Friedrich U, Schwab M, Griese E-U, Fritz P, Klotz U 2001 Telomeres in neonates: new insights in fetal hematopoiesis. Pediatr Res 49:252-256

18. Jeanclos E, Krolewski A, Skurnick J, Kimura M, Aviv H, Warram JH, Aviv A 1998 Shortened telomere length in white blood cells of patients with IDDM. Diabetes 47:482-486

19. Samani NJ, Boutby R, Butler R, Thompson JR, Gordal AH 2001 Telomere shortening in atherosclerosis. Lancet 358:472-474 